\title{
ARTICLE Colonic epithelial mTORC1 promotes ulcerative colitis through COX-2-mediated Th17 responses
}

\author{
Xiaojun Lin ${ }^{1}$, Qiuyi Sun ${ }^{1}$, Ling Zhou ${ }^{2}$, Minhong $\mathrm{He}^{2}$, Xiaoying Dong ${ }^{2}$, Mingqiang Lai ${ }^{1}$, Miao Liu ${ }^{1}$, Yongchun Su ${ }^{1}, \mathrm{Chunhong}{ }^{1}{ }^{1}$,
} Zelong $\mathrm{Han}^{3}$, Side Liu ${ }^{3}$, Hang Zheng ${ }^{2}$, Yu Jiang ${ }^{4}$, Hui Ling ${ }^{5}$, Mangmang Li ${ }^{1}$, Juan Chen ${ }^{1}$, Zhipeng Zou ${ }^{1}$ and Xiaochun Bai ${ }^{1}$

The functional role of colonic epithelium in the pathogenesis of ulcerative colitis (UC) remains unclear. Here, we reveal a novel mechanism by which colonic epithelia recruit T helper-17 (Th17) cells during the onset of UC. mTOR complex 1 (mTORC1) was hyper-activated in colonic epithelia of UC mice. While colonic epithelial TSC1 (mTORC1 negative regulator) disruption induced constitutive mTORC1 activation in the colon epithelia and aggravated UC, RPTOR (essential mTORC1 component) depletion inactivated mTORC1 and ameliorated UC. TSC1 deficiency enhanced, whereas RPTOR ablation reduced the expression of cyclooxygenase 2 (COX-2), interleukin-1 (IL-1), IL-6, and IL-23, as well as Th17 infiltration in the colon. Importantly, inhibition of COX2 reversed the elevation in the expression of these proinflammatory mediators induced by TSC1 deficiency, and subsequently reduced the symptoms and pathological characteristics of UC in mouse models. Mechanistically, mTORC1 activates COX-2 transcription via phosphorylating STAT3 and enhancing it's binding to the COX-2 promoter. Consistently, enhanced mTORC1 activity and COX2 expression, as well as strong positive correlation between each other, were observed in colonic epithelial tissues of UC patients. Collectively, our study demonstrates an essential role of epithelial mTORC1 in UC pathogenesis and establishes a novel link between colonic epithelium, Th17 responses, and UC development.

Mucosal Immunology (2018) 11:1663-1673; https://doi.org/10.1038/s41385-018-0018-3

\section{INTRODUCTION}

Inflammatory bowel disease (IBD), which mainly comprises ulcerative colitis (UC) and Crohn's disease (CD), is a global disease with increasing prevalence. ${ }^{1}$ Although its pathogenesis remains unclear, IBD is believed to primarily result from a failure in the homeostasis of innate and adaptive immune responses including Th2 and Th17 cells. ${ }^{2}$ It was reported that the inflammatory intestine of UC patients was characterized by massive Th17 cells infiltration and resulting amplification of cytokine release and inflammatory processes. ${ }^{3,4}$ In humans, Th17 cells are initially induced by the synergism between TGF- $\beta$ and IL- $6,{ }^{5}$ followed by an expansion stage directed by IL-21 activation ${ }^{6}$ and a stabilization phase driven by the pro-inflammatory cytokines IL-1 and IL-23. ${ }^{7,8}$ However, the cell types and upstream pathways initiating this cytokines-synergized Th17 response in UC remain unclear.

The mechanistic target of rapamycin (mTOR) is a serine/ threonine kinase that exists as the catalytic subunit of two biochemically distinct complexes called mTOR complex 1 (mTORC1) and mTORC2. Classically, mTORC1 controls cell autonomous growth in response to nutrient availability and growth factors. Recent evidence revealed the involvement of immune cell-specific mTORC1 in various inflammatory diseases. $^{9-11}$ It has also been shown that whole body mTORC1 inhibition reduces inflammation and consequent severity of experimental UC. ${ }^{12-14}$ However, the functional role and the underlying mechanism of mTORC1 activation in the development of UC are currently unknown. Particularly, experimental evidence on the contribution of cell type-specific mTORC1 to the initiation of Th17 responses and pathogenesis of UC is lacking.

Colonic epithelial cells are the cell boundary between the external environment and tissues of the gastrointestinal tract. Prior to the recruitment of inflammatory cells including macrophages, lymphocytes and neutrophils, innate signaling within the colonic epithelial cells is crucial for initiating the early inflammatory responses during the onset of UC. To explore the potential importance of colonic epithelial mTORC1 signaling in UC development, we constructed two reciprocal gene knockout mouse models: (1) colonic epithelium-deletion of TSC1, a key negative upstream regulator of $\mathrm{mTORC1}$, leading to constitutive mTORC1 activation, and (2) colonic epithelium-deletion of RPTOR, leading to permanent mTORC1 deactivation. ${ }^{15}$ Together with clinical samples, we demonstrated high activity and essential role of colonic epithelial mTORC1 in the development of UC. Importantly, we established a novel link between colonic epithelium and Th17 cells recruitment during UC through the mTORC1-STAT3-COX2 pathway.

\footnotetext{
${ }^{1}$ Department of Cell Biology, School of Basic Medical Science, Southern Medical University, 510515 Guangzhou, China; ${ }^{2}$ Department of Oncology, Nanfang Hospital, Southern Medical University, 510515 Guangzhou, China; ${ }^{3}$ Department of Gastroenterology, Nanfang Hospital, Southern Medical University, 510515 Guangzhou, China; ${ }^{4}$ Department of

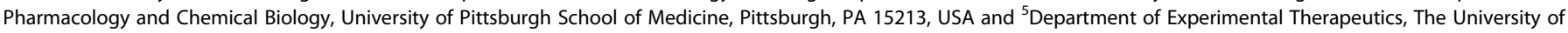
Texas MD Anderson Cancer Center, Houston, TX 7054, USA

Correspondence: Juan Chen (landj@smu.edu.cn) or Zhipeng Zou (zzp@smu.edu.cn) or Xiaochun Bai (baixc15@smu.edu.cn)

These authors contributed equally: Xiaojun Lin, Qiuyi Sun, Ling Zhou.
}

Received: 11 May 2017 Revised: 29 January 2018 Accepted: 17 February 2018

Published online: 6 August 2018 


\section{RESULTS}

mTORC1 is hyper-activated in the colonic epithelium of UC patients and UC mouse model and essential for UC development To test the functional importance of MTORC1 in UC, we mimicked the disease of human UC using the well-established mouse model with DSS induction and examined the activity of mTORC1 in colonic epithelium of the model. To study the relevance of mTORC1 in DSS-induced UC, we isolated colonic epithelia and measured the activities of mTORC1 by western blotting and immunohistochemistry. The level of phosphorylated S6 (S235/ 236), one of the best characterized substrates of mTORC1, but not that of phosphorylated Akt (S473), the well-characterized target of mTORC2, was significantly increased in colon tissues from DSStreated mice in comparison with the control group (Fig. 1a, left and right). Flow cytometry assay for CD326 confirmed the purity of the colonic epithelia (92.5\%) (Figure S1). Consistently, oral administration of rapamycin markedly alleviated the colonic inflammatory response of DSS-induced UC, as reflected by the recovery of body weight (Fig. $1 \mathrm{~b}$ ) and colon length (Fig. 1c), as well as reduced histopathological impairments (Fig. 1d) and disease activity index (DAI) score (Fig. 1e). The efficacy of oral rapamycin administration in specific mTORC1 inhibition was confirmed by the dramatic reduction of S6 (S235/236) phosphorylation, but not Akt phosphorylation (S473), in colon epithelia following the rapamycin treatment (Fig. 1a). Concordant with the above findings, higher $\mathrm{S} 6$ phosphorylation was observed in colon epithelia of human UC patients $(n=17)$ comparing with that of the control human subjects $(n=7)$ (Fig. 1f). In addition, S6 phosphorylation was positively correlated with clinical activity index (Spearman $R=0.6827 ; P=0.0002$ ) (Fig. 1g). Taken together, these results demonstrate that colonic epithelial mTORC1 becomes activated in UC of both human and mice and is correlated with disease activity. Reversely, rapamycin inhibits mTORC1 and consequently reduces the severity of experimental UC.

\section{Generation of colon epithelia-specific TSC1 or RPTOR knockout} mice

To investigate the functional role of colonic epithelial mTORC1 in UC, we generated mice with specific knockout of TSC1 or RPTOR gene in colonic epithelium by crossing homozygous $T S C 1^{\mathrm{FL} / \mathrm{FL}}$ or $R P T O R^{\mathrm{FL} / \mathrm{FL}}$ mice with transgenic mice expressing Cre recombinase under the control of the colonic epithelial cell(CEC)-specific carbonic anhydrase 1(CA1) promoter (see Figure S2A for schematic diagram of the knockout mouse generation). ${ }^{16}$ Immunoblot analysis (Figure S2B) of epithelial extracts confirmed the depletion of targeted genes, and the consequent alterations of S6 phosphorylation in TSC1 knockout or RPTOR knockout mice, respectively. The observed residual TSC1 or Raptor protein expression in colonic epithelia in the knockout mice might reflect incomplete recombination mediated by the Cre recombinase.

mTORC1 in the colonic epithelium is dispensable for normal colon function and development

To examine whether the absence of colonic epithelial TSC1 or $R P T O R$ results in defective colonic function, we analyzed colon length, histological structure and proliferative activity in colon tissue, as well as the numbers and distribution of secretory cells in colonic epithelium-specific TSC1 knockout (KO) mice or RPTOR KO mice. No significant difference in colon length was observed between both types of $\mathrm{KO}$ mice and their control littermates (Figure S2A). Additionally, hematoxylin-eosin (HE) staining revealed no significant differences in histological structure (Figure S3B), numbers of colonic epithelial cells and mucous cells, and the thickness of the colonic mucosa (Figure S3C) between KO mice and their littermates. Periodic Acid-Schiff (PAS) and ki67 staining further showed that both numbers and distribution pattern of secretory cells and proliferating cells were similar between these
KO mice and their control littermates (Figure S3B). Collectively, these results suggest that mTORC1 activity in the colonic epithelium is not essential for normal colonic function and development.

\section{Colonic epithelium-specific mTORC1 alterations affect the} pathogenesis of UC

We next tested the role of mTORC1 activity in DSS-induced UC with the generated knockout mouse models. In the experimental condition of DSS stimulation, colonic epithelium-specific TSC1 depletion resulted in more weight loss (Fig. 2a), colon shortening (Fig. 2b), histopathological impairment (Fig. 2c), as well as reduction in secretory (Figure S4A) and proliferating cells (Figure S4B) comparing with the control littermates. In addition, a significant enhancement of S6 phosphorylation (S235/236), but not Akt phosphorylation (S473), was observed in TSC1 KO mice compared to that of control mice (Figure S5A left, Figure S5B). This suggests that mTORC1 but not mTORC2 was activated in the epithelia of TSC1 KO mice. By contrast, colonic epithelium-specific mTORC1 disruption (RPTOR depletion) led to a reduction of weight loss (Fig. 2e), restored colon length (Fig. 2f), alleviated the histopathological impairment (Fig. 2g), as well as restored secretory (Figure S4A) and proliferating cells (Figure S4B) in the experimental condition of DSS induction, comparing with the control mice. In addition, S6 phosphorylation (S235/236), but not Akt phosphorylation (S473), was significantly reduced in the colonic epithelium of RPTOR KO mice compared to that of control mice (Figure S5A right, Figure S5B). Taken together, these results from two opposite genetic mice models, namely, colonic epithelium-specific TSC1 and RPTORKO mice, suggest that the severity of experimental UC is dependent on colonic epithelial mTORC1 activity.

\section{mTORC1 activates inflammatory pathways that leads to Th17} infiltration

To clarify the underlying mechanism of mTORC1 involvement in UC, the colonic epithelial mRNA expression profiles of TSC1 KO mice were compared to those of their littermates after DSS stimulation (Fig. 3a). The expression of 951 mRNAs was significantly enhanced, whereas that of 858 mRNAs was reduced ( $\geq 3$-fold), in the TSC1 KO mice. These mRNAs were functionally categorized into multiple classes by KEGG Pathway mapping, including the Toll-like receptor signaling pathway, cytokine-cytokine receptor interaction, the chemokine signaling pathway, the Jak-STAT signaling pathway, and arachidonic acid metabolism. The expression of several critical genes in development of UC was dramatically upregulated in the colonic epithelium of mice with TSC1 ablation. Of particular interest, IL-6 (23.8-fold), IL-1A (20.8-fold), IL-12B (11.9-fold), IL-23A (7.4-fold), IL22 (5.2-fold) and Cyclooxygenase 2 (COX-2) (25.8-fold) (Fig. 3b) were closely linked to each other and may constitute a critical signaling axis. For instance, COX-2 can be induced by IL-1 and IL$6,{ }^{17-19}$ and conversely, IL-6 secretion can be induced by COX-2/ Prostaglandin E2 (PGE2). ${ }^{20,21}$ Furthermore, secretion of IL-23 (made up of IL-12B and IL-23A) ${ }^{22}$ can be significantly promoted by IL-1/IL-6, and the IL-23/Th17 axis can be stabilized and potentiated by PGE2. ${ }^{23,24}$ Thus, based on previous knowledge that the Th17 response is induced, expanded, and stabilized by the synergistic action of IL-1, IL-6, IL-22, and IL-23, we focused our subsequent functional studies on this critical axis. The induction or reduction of cytokines, including IL-1A, IL-6, and IL-23A at the mRNA level in the TSC1 or RPTOR ablated colonic epithelium, respectively, was confirmed by quantitative reverse transcription PCR (RT-qPCR) (Fig. 3c). COX-2 expression at the protein level was nearly undetectable in colon tissues from normal mice, moderately increased in the colonic epithelium of mice with experimental UC, and dramatically enhanced or reduced in the colonic epithelium of TSC1 or RPTOR KO mice, respectively (Fig. 3d). To test 
a
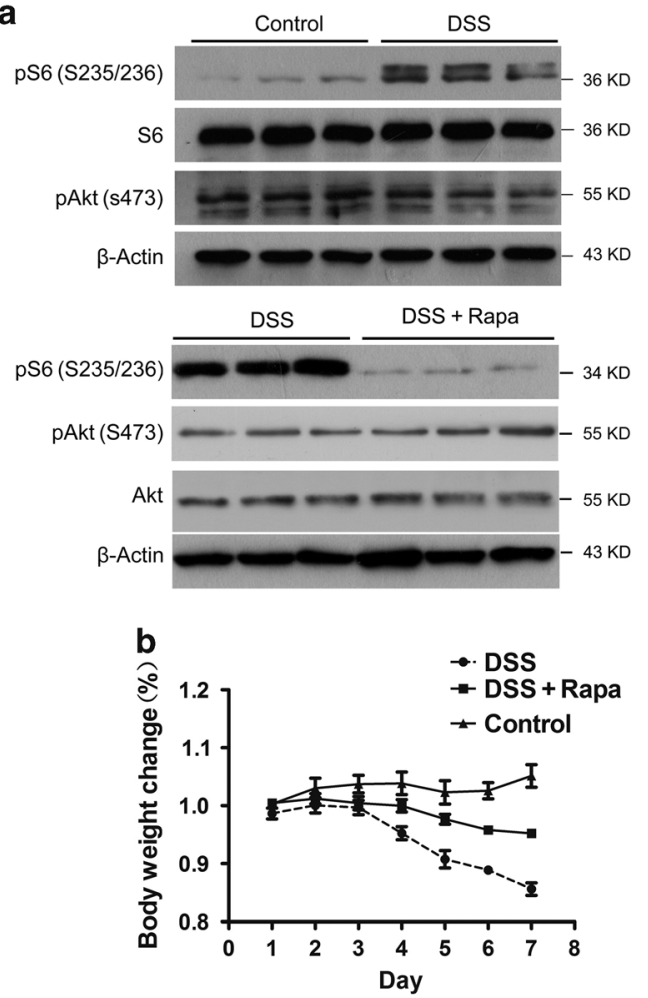

d

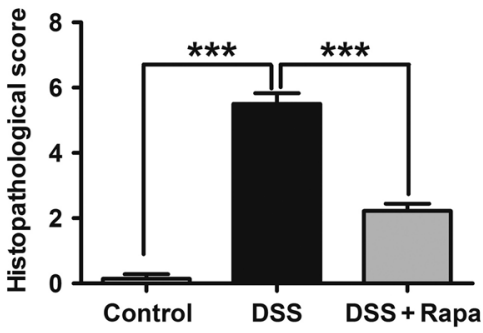

Control

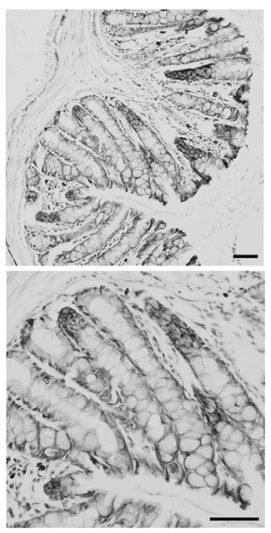

DSS

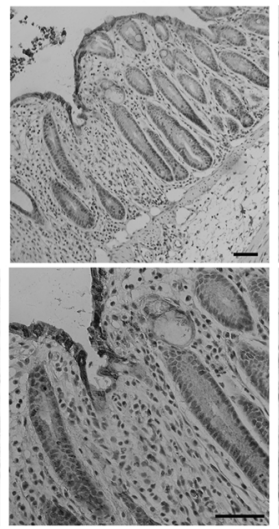

DSS + Rapa

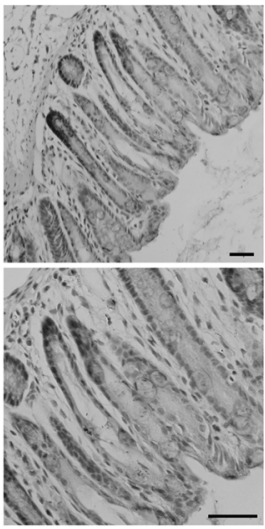

e

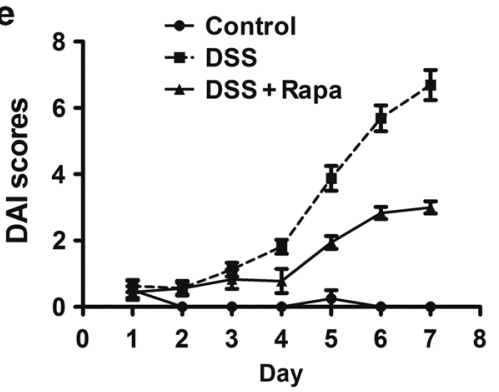

f

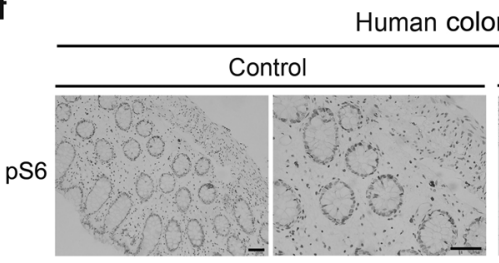

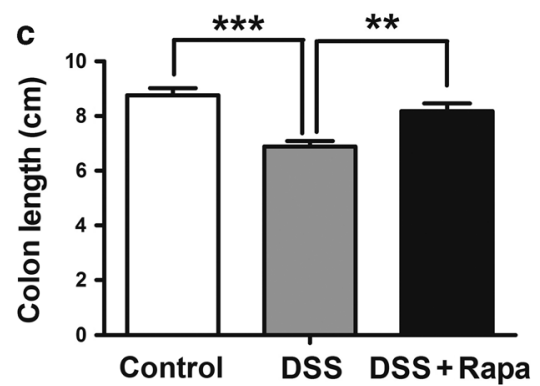

$(235 / 236)$

g

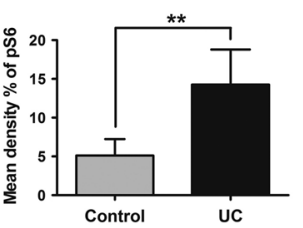

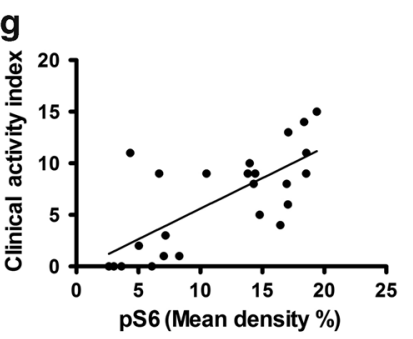

Fig. 1 mTORC1 is activated in human UC and DSS-induced experimental UC and essential for UC development. a Phosphorylated S6 (S235/ 236) in colonic epithelial cells and colonic sections of control, DSS-treated mice, and DSS-treated mice with rapamycin administration was monitored using western blotting (left) and immunohistochemical staining (right), respectively. b Changes in body weight (percentage of original body weight) over time (days) in control, DSS-treated mice, and DSS-treated mice with rapamycin administration $(n=6-8)$. c Comparison of colon length between these mice. ${ }^{* *} P<0.01 ;{ }^{* *} P<0.0001(n=6-8)$. d Histopathological score and e disease activity index score of these mice. ${ }^{* *} P<0.0001(n=6-8)$. f S6 phosphorylation (S235/236) in colon biopsies from control subjects or patients with ulcerative colitis (UC) was observed via immunohistochemical staining. ${ }^{* *} P<0.01$. g Correlation between S6 phosphorylation (S235/236) in colon biopsies and clinical activity index. Scale bar, $50 \mu \mathrm{m}$

whether COX-2, IL-1, IL-6, and IL-23 modulated by mTORC1 were associated with enhanced Th17 cell infiltration in the colon, we labeled lamina propria Lymphocytes with both anti-CD4 and antiRORyt antibodies, and labeled colon sections with anti-CD4 and anti-IL-17 antibodies. The resultant RORyt+ CD4+ cells and IL-17 producing CD4+ cells (both representing Th17 cells) were visualized using both flow cytometry (Fig. 3e) and confocal microscopy (Figure S6), respectively. Consistent with our hypothesis, TSC1 KO triggered a marked influx of Th17 cells, whereas RPTOR KO led to a significant reduction of such cells in lamina propria of colon tissues of mice with UC. Finally, no significant difference in segmented filamentous bacteria (SFB) copy number was observed in colon tissue of these mice (Figure S7), suggesting equally colonization of them by SFB and excluding the possibility 


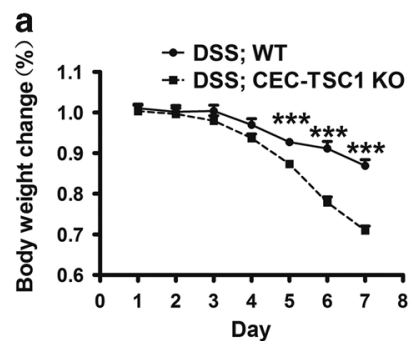

b

DSS; WT

DSS; CEC-TSC1 KO

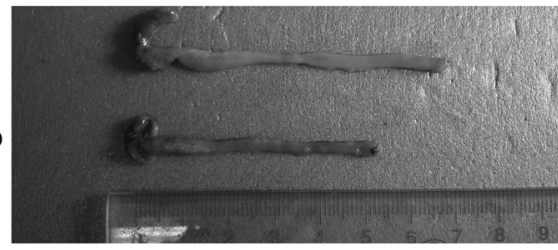

DSS

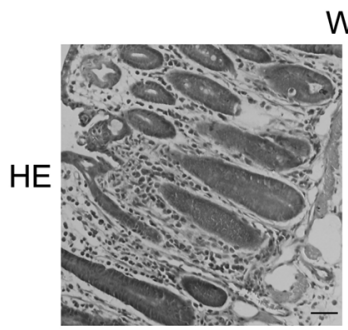

WT
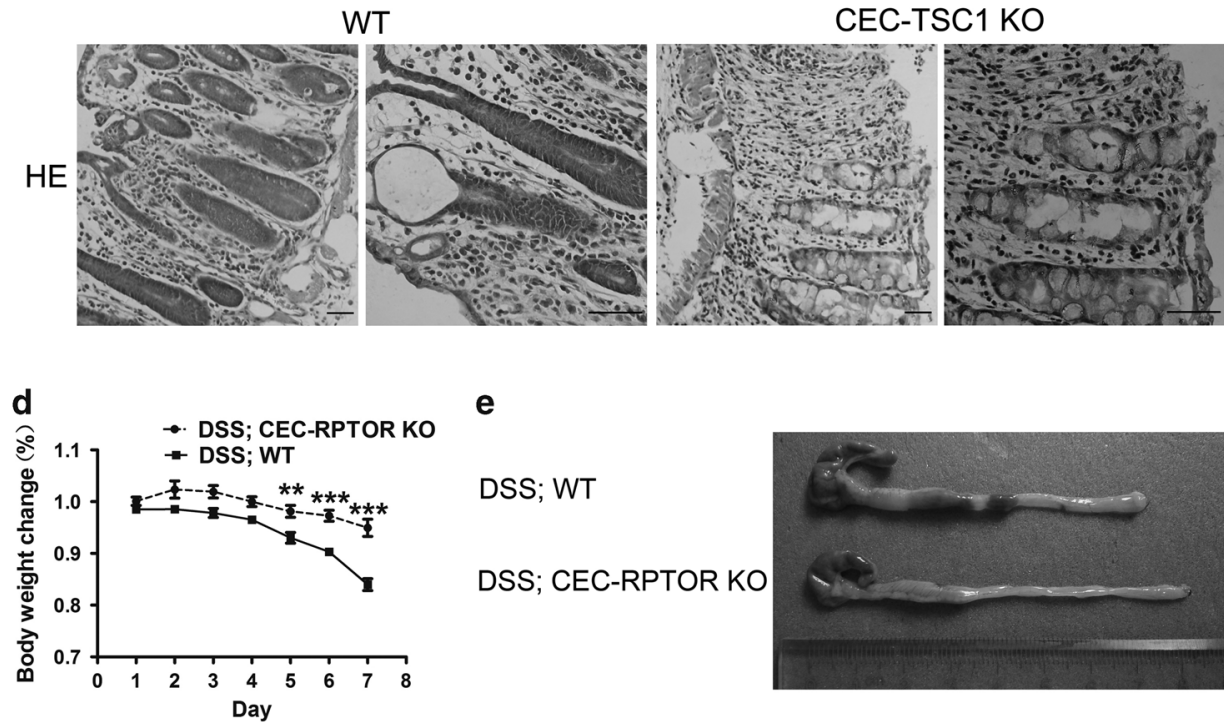

e

DSS; WT

DSS; CEC-RPTOR KO

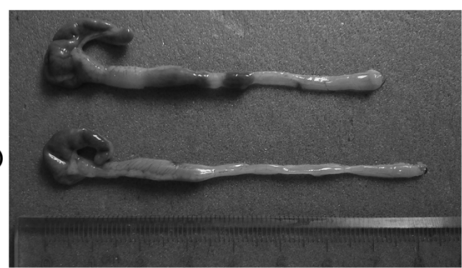

f

DSS

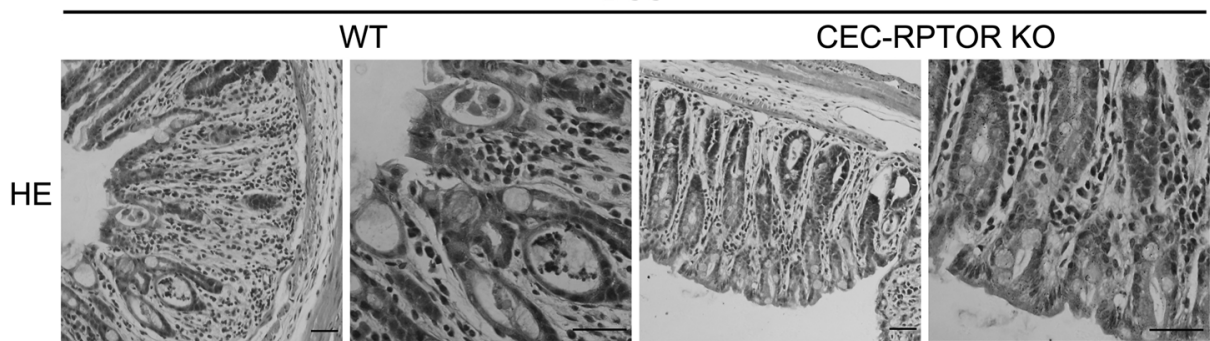

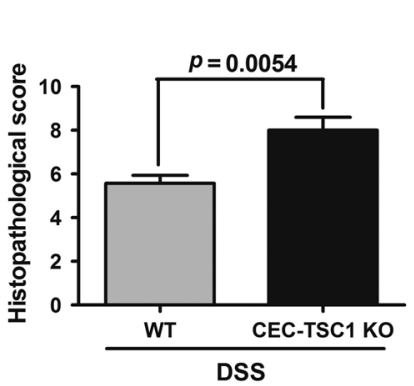
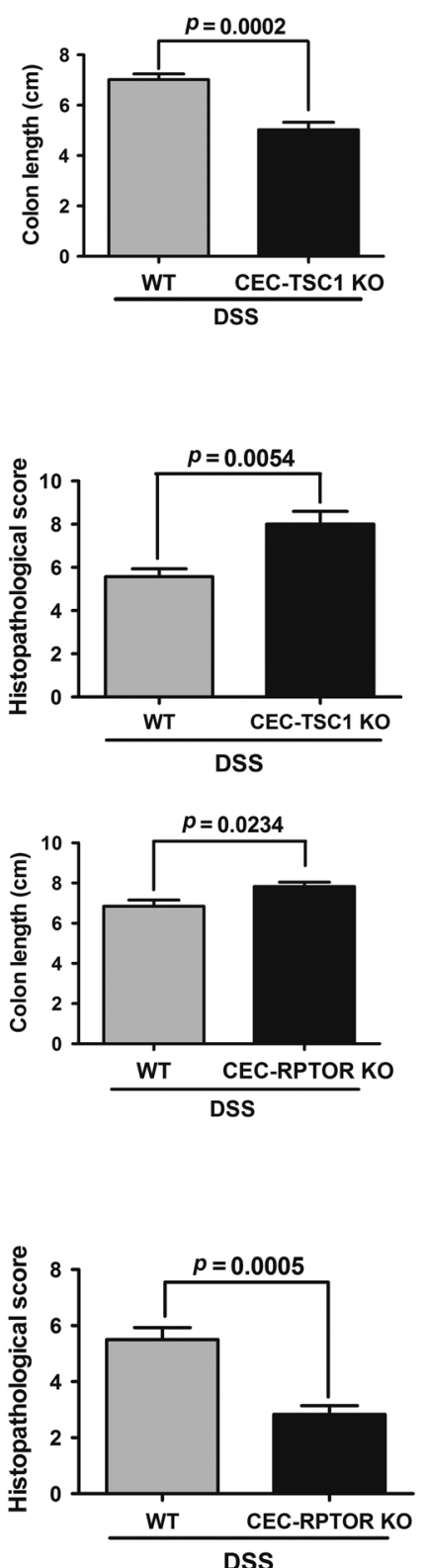

Fig. 2 Colonic epithelium-specific mTORC1 activation exacerbates, whereas mTORC1 disruption alleviates DSS-induced UC. a, d Changes in body weight (percentage of original body weight) over time (days) in mice with colonic epithelium-specific TSC1 (a) or RPTOR (d) ablation treated with DSS for 7 days. ${ }^{* *} P<0.01 ;{ }^{* *} P<0.0001(n=6-8)$. b, e Gross morphology (left) and statistical analysis of the length (right) of the large intestine in the indicated mice $(n=6-8)$. c, $\mathbf{f}$ Left, Hemotoxyline-Eosin (HE) staining of colon sections from the indicated mice. Original magnification, $\times 40$. Scale bar, $50 \mu \mathrm{m}$. Right, histopathological score of these mice $(n=6-8)$. CEC-TSC1 KO or CEC-RPTOR KO, TSC1 or RPTOR knockout in colonic epithelial cells; WT, control

that mTORC1-mediated Th17 induction is through SFB, as reported recently. ${ }^{25}$

mTORC1 activates inflammatory response by upregulation of COX-2 expression

Based on previous findings that both IL- 6 secretion and the IL23/Th17 axis can be induced by PGE2, one of the most important products of COX-2, ${ }^{20,23}$ we hypothesized that mTORC 1 activity in the colonic epithelium promotes experimental UC via COX-2. In accordance with this hypothesis, oral administration of the COX2 specific inhibitor celecoxib markedly ameliorated UC in mice with TSC1 ablation, but not in mice without TSC1 ablation, as evidenced by the prevention from loss of body weight (Fig. 4a), inhibition of the shortening of the colon (Fig. 4b), and reduction in histopathological damage (Fig. 4c). Accordingly, celecoxib administration reversed the upregulation of IL-1, IL-6, and IL-23 in the colonic epithelium (Fig. 4d) and the corresponding Th17 influx in mice with TSC1 ablation (Fig. 4e and S1). Since a recent report suggested an important role for colonic epithelial SAA in regulating intestinal Th17 recruitment, ${ }^{25}$ SAA mRNA level in colonic epithelia from control, TSC1 KO, or RPTOR KO mice with UC were analyzed by RT-QPCR and no significant difference was observed (Figure S9), suggesting different mechanism may be involved in Th17 response mediated by SFB colonization or mTORC1 activity. Collectively, these results suggest that colonic epithelial mTORC1 promotes the upregulation of IL-1/IL-6/IL-23 and Th17 infiltration via COX-2, at least partially. 


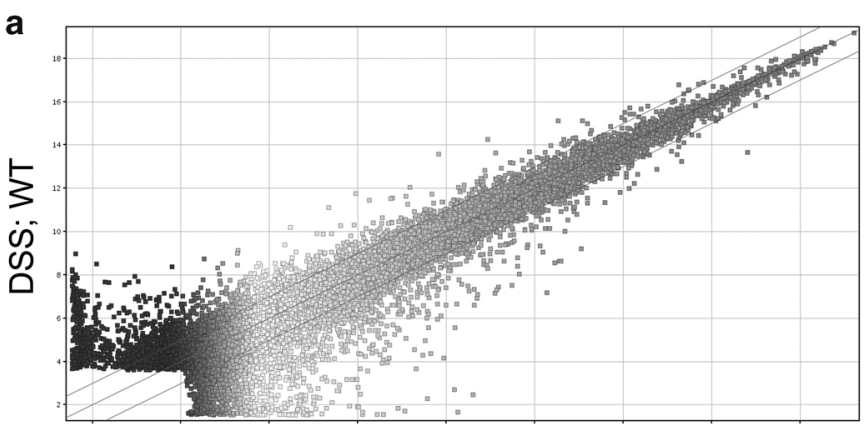

DSS; CEC-TSC1 KO
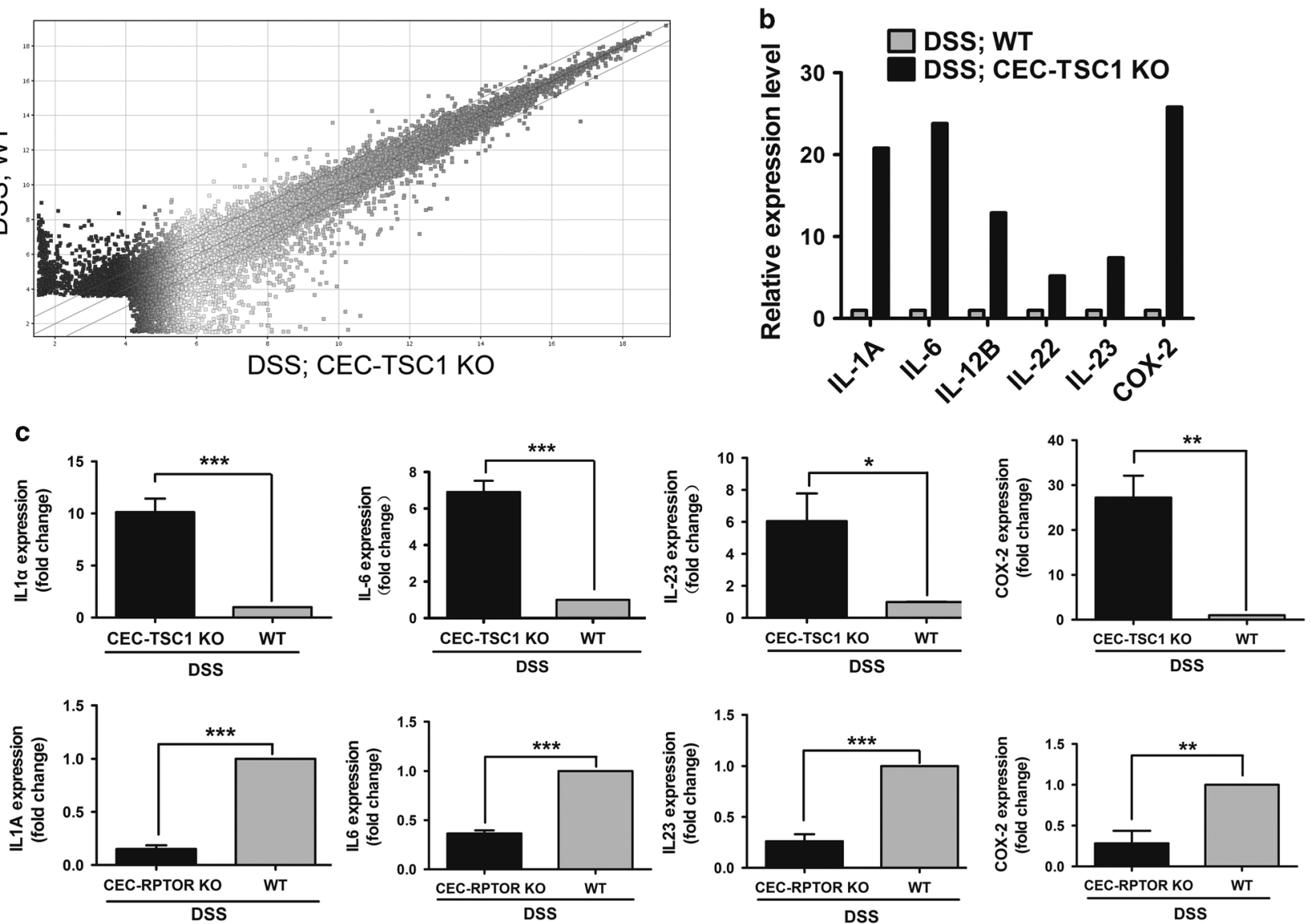

d
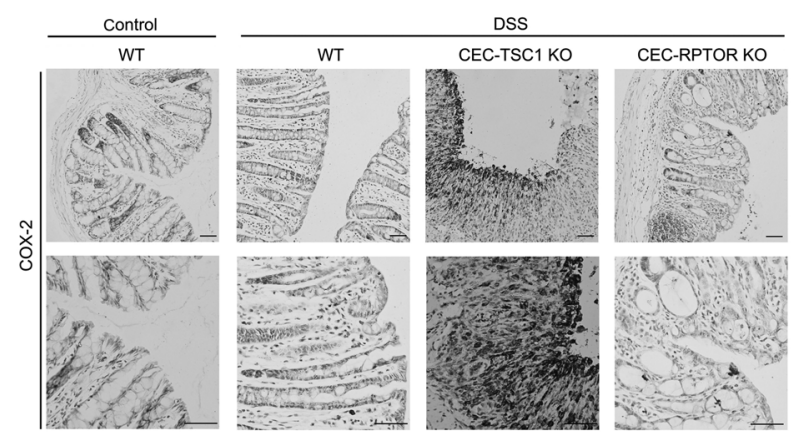

e
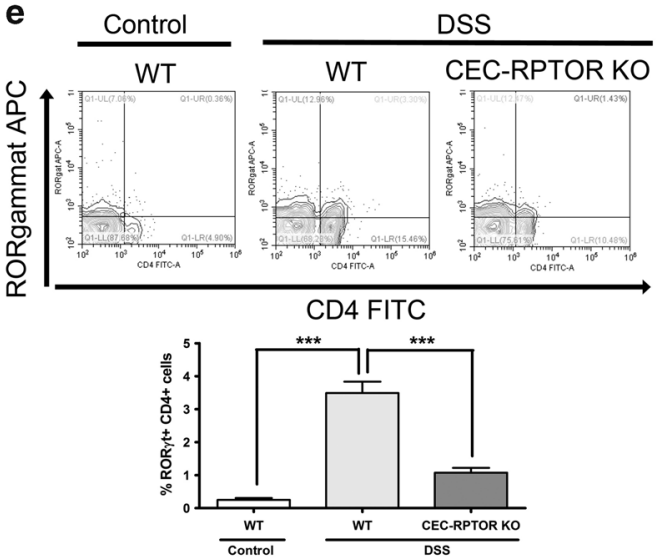

Fig. 3 mTORC1 activity in the colonic epithelium is critical for the regulation of the IL-23-Th17 axis. a Control mice (WT) or mice with TSC1 knockout in colonic epithelial cells (CEC-TSC1 KO) were treated with DSS, and after 7 days, total RNAs from colonic epithelial cells (CEC) were analyzed by microarray. Differential expression patterns of mRNAs between these groups are shown using a matrix plot. $\mathbf{b}$ Comparison of the relative expression level of multiple inflammatory factors essential for the IL-23-Th17 axis in CEC between control or CEC-TSC1 KO mice. c Verification of IL-1A, IL-6, IL-23A, and COX-2 upregulation or downregulation at mRNA level in CEC from mice with TSC1 or RPTOR ablation in colonic epithelial cells (CEC-TSC1 KO or CEC-RPTOR KO), respectively, using quantitative reverse transcription PCR (RT-qPCR). ${ }^{*} P<0.05 ;{ }^{* *} P<$ 0.01 ; ${ }^{* *} P<0.0001(n=6-8)$. d Verification of COX-2 protein upregulation or downregulation at the tissue level in CEC from CEC-TSC1 KO or CEC-RPTOR KO mice, respectively, using immunohistochemical assays. Original magnification, $\times 20$ and $\times 40$ as indicated by the scale bars. Scale bar, $50 \mu \mathrm{m}$. e control mice (WT) and mice with RPTOR deletion in colonic epithelial cells (CEC-RPTOR KO) were treated with DSS. 7 days post-treatment, mice were sacrificed and mononuclear cells of LP were isolated and labeled with anti-CD4 and anti-RORyt sequentially, followed by visualization of CD4 and ROR $\gamma$ t labeling via flow cytometry. CD4+ lymphocytes were further checked for ROR $\gamma \mathrm{t}$ labeling

mTORC1 upregulates COX-2 expression via a STAT3-dependent mechanism

We next moved on to determine the mechanism underlying mTORC1-induced upregulation of COX-2. Based on previous findings that STAT3 activates COX-2 transcription, ${ }^{26}$ and mTORC1 directly phosphorylate STAT3 at $\mathrm{S} 727,{ }^{27}$ we hypothesized that
STAT3 might be the factor bridging mTORC1 activity and COX2 expression. We first evaluated phosphorylation of STAT3 at S727 on colonic sections by immunohistochemistry. As expected, STAT3 S727 phosphorylation was activated in the epithelia of DSS treated mice, which was further enhanced with TSC1 ablation (Fig. 5a). Consistently, with in vitro cell model 


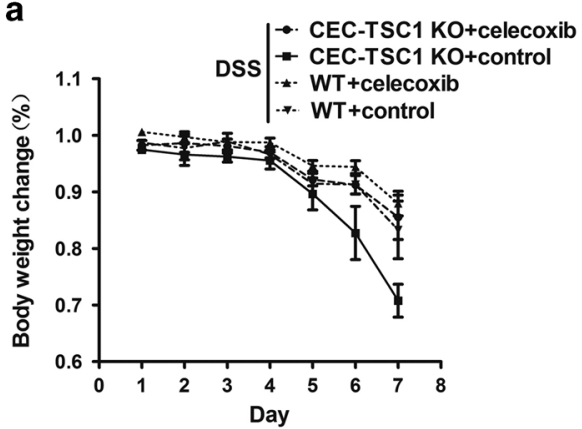

C
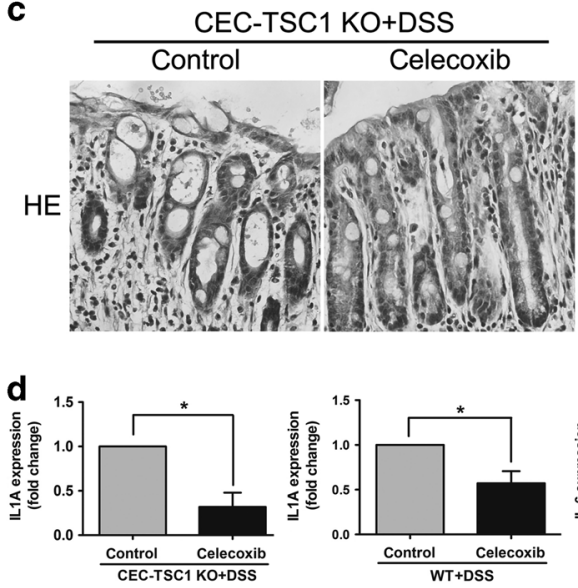

e
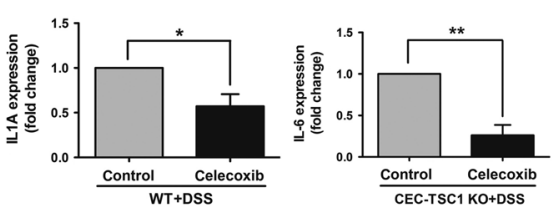

CEC-TSC1 KO+DSS
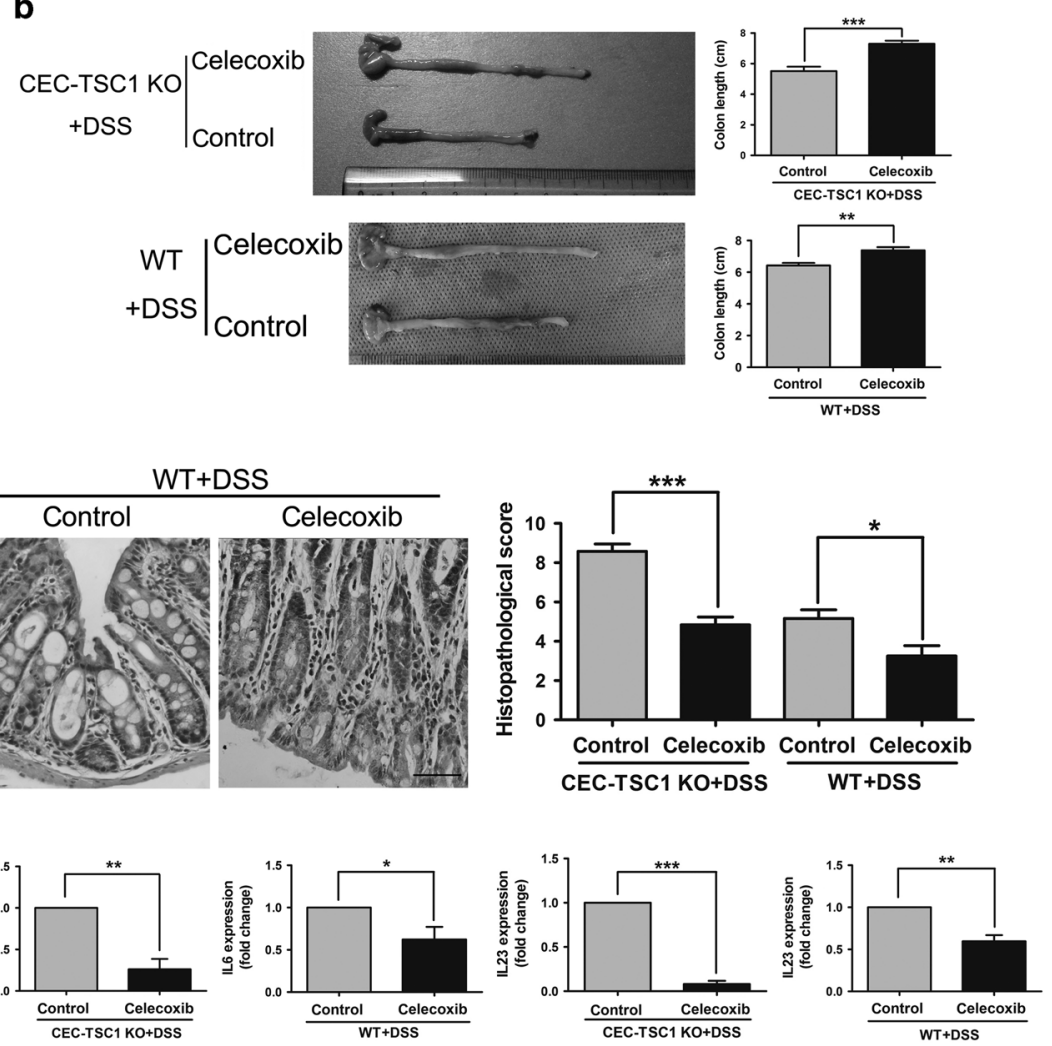

WT+DSS
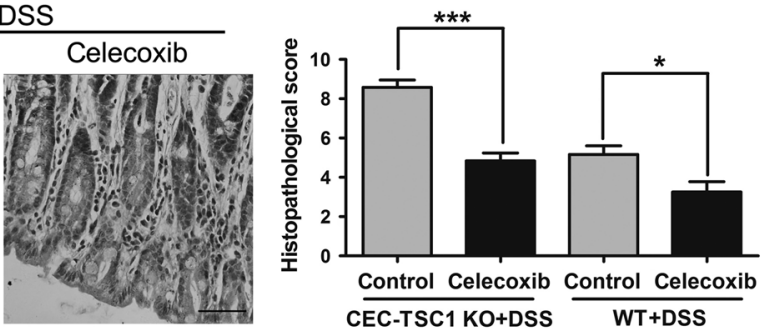

WT+DSS

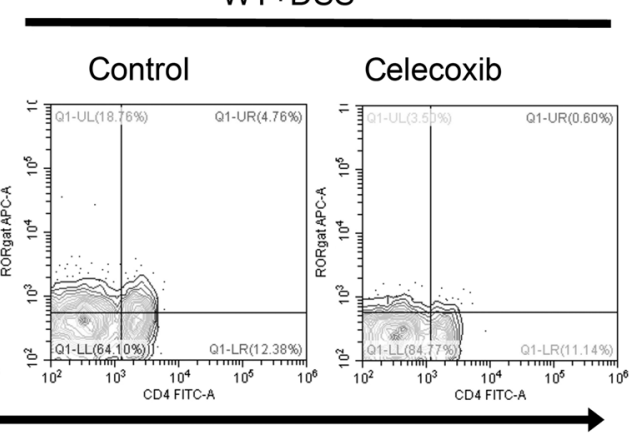

CD4 FITC

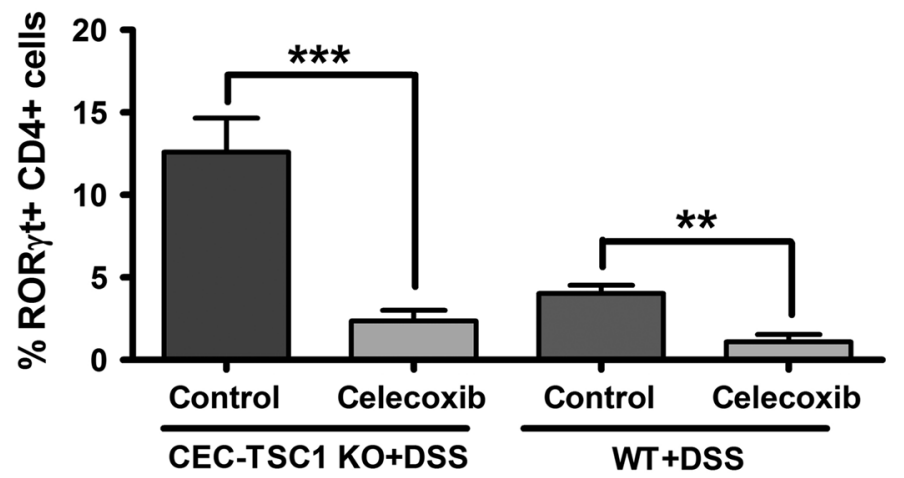

we found that TSC1 depletion enhanced STAT3 S727 phosphorylation in NCM460 colonic epithelial cells, both in the presence and absence of lipopolysaccharide (LPS), a key factor in colitis inducing COX-2 expression (Fig. 5b). The induction of COX-2 expression by TSC 1 depletion was abrogated by simultaneous STAT3 depletion (Fig. 5c). Additionally, the binding of STAT3 to COX-2 promoter induced by LPS was dramatically reduced upon mTORC 1 inhibition by rapamycin treatment (Fig. 5d). Collectively, these results suggest that STAT3 is required for mTORC1-directed COX-2 expression, possibly 
Fig. 4 mTORC1 activity in the colonic epithelium promotes UC via COX-2. Control mice (WT) or mice with TSC1 ablation in colonic epithelial cells (CEC-TSC1 KO) were treated with DSS and celecoxib as described in the materials and methods section. a Changes in body weight (percentage of the original body weight) over time (days) of the indicated mice. $\mathbf{b}$ Comparison of colon length between the indicated mice. Left, representative image showing the gross morphology of the large intestine from the indicated mice. Right, statistical analysis of colon length. ${ }^{* *} P<0.0001(n=6-8)$. c Left, Hemotoxyline-Eosin (HE) staining of colon sections from the indicated mice. Right, histopathological score of these mice. ${ }^{* *} P<0.0001(n=6-8)$. d Analysis of IL-1, IL-6 and IL-23 expression at the mRNA level in colonic epithelial cells (CEC) of the indicated mice. ${ }^{*}, P<0.05 ;{ }^{*} P<0.01(n=6-8)$. e The indicated mice were sacrificed and mononuclear cells of LP were isolated and labeled with anti-CD4 and anti-RORyt sequentially, followed by visualization of CD4 and ROR $\gamma$ t labeling via flow cytometry. CD4+ lymphocytes were further checked for ROR $\gamma$ t labeling

through its binding to COX-2 promoter after being phosphorylated by mTORC1.

mTORC1 activity is positively correlated with COX-2 expression in clinical UC samples

To verify the relevance of mTORC1-COX2 signaling axis in human UC, we analyzed by immunohistochemistry colonic biopsies from 7 normal subjects and 17 UC patients for S6 phosphorylation and COX2 expression. Both 56 phosphorylation and COX2 expression were markedly enhanced in colon tissues (including colonic epithelium) of human UC patients compared to those in normal subjects (Figs. 6a, b, Table S2). Additionally, S6 phosphorylation and COX2 expression were strongly positively correlated (Spearman $R=0.6572 ; P=0.0005$; Pearson $R=0.6166 ; P=0.0013$ ) in all of the enrolled subjects including both healthy people and UC patients (Fig. 6c). Taken together, these results highlight the indispensable role of the mTORC1-COX2 signaling axis in the etiology of human UC.

\section{DISCUSSION}

In the present study, with two opposite genetic mice models leading to constitutive mTORC1 activation or deactivation, respectively, we proved the essential role of mTORC1 activity in colonic epithelium in UC pathogenesis. Colonic epitheliumspecific mTORC1 activity is not essential for mouse development and normal colon function, but is critical for DSS-induced UC, providing a new rationale for targeting colonic-epithelial mTORC1 as a highly effective and safe strategy to prevent and/or treat UC. Our study also revealed a novel mechanism of activation of Th17 cell recruitment in colon tissue during the onset of UC by colonic epithelium-specific mTORC1-STAT3-COX-2 signaling.

While mTORC1 inhibitors such as rapamycin, P2281, and mTOR kinase inhibitor AZD8055 have been shown to be potentially effective in alleviating experimental UC by reducing leukocyte sticking and extravasation, or by balancing Th1/Th17/Treg Profile, ${ }^{12-14}$ evidence on the functional importance of colonic epithelial-specific mTORC1 in the development of UC is currently lacking. Since mTORC1 inhibitors act globally without tissueselectivity, deciphering the function of mTORC 1 in specific tissue types using these inhibitors is difficult. Hence, a genetic mice model with tissue-specific disruption of mTORC1 is essential to elucidate the role of this molecule in UC in vivo. Combining the results from epithelium-specific ablation of both TSC1 and RPTOR, we reciprocally substantiated that colonic-epithelial mTORC1 activity is both sufficient and essential for DSS-induced Th17 recruitment and UC development.

COX-2, an inducible COX in response to inflammatory initiators such as interleukin-1 and tumor necrosis factor, ${ }^{28,29}$ has been suggested to be involved in UC development based on the findings that COX-2 inhibition reduces inflammation and the severity of experimental UC by suppressing PGE , IL-1 , and NOS activity, and by reducing neutrophil infiltration. ${ }^{30}$ However, the signaling networks that govern COX2 activity and its downstream effects in pathogenesis of UC are not clear. Our study indicates that the pathway by which colonic epithelium-specific activation of mTORC1 promotes UC may include the STAT3-dependent activation of COX-2 transcription. Although mTORC1 directly phosphorylates STAT3 in vitro, as shown by our previous study, ${ }^{27}$ possibility of feedback activation of STAT3 by mTORC1 via IL- $22^{25}$ cannot be excluded, since our microarray data demonstrated markedly induction of colonic epithelial IL-22 in response to mTORC1 over-activation (Fig. 3b). The functional role of COX-2 in UC is ultimately dependent on its products - prostanoids. Prostanoids produced via COX-2 contribute positively to inflammation, pain, and fever. ${ }^{31}$ Consistently, the COX-2 inhibitors celecoxib and rofecoxib demonstrated beneficial effects for the treatment of UC patients in a clinical trial $^{32}$ and experimental UC rodents. ${ }^{30,33}$ PGE2, a major product of COX2, can actually act, depending on its level, as a proinflammatory mediator in IBD. High levels of PGE2 may activate EP2/EP4 receptors and worsen IBD by shifting the IL-12/IL-23 balance in favor of IL-23 on dendritic cells (DCs), which promotes Th17 cell differentiation and further exacerbates the inflammatory response. ${ }^{23,24}$ Consistent with these findings, our results showed that in vivo administration of celecoxib leads to a substantial downregulation of IL-1, IL-6, and IL-23 in the colonic epithelium and a prominent reduction of Th17 cell infiltration in colon tissues of TSC1 KO mice, suggesting the proinflammatory effects of COX-2 abnormally activated by mTORC1 in UC may be through recruitment of Th17 cells, at least partially. The contradict role of COX-2 in the protection of intestinal barrier ${ }^{34}$ may be ascribed to the binding of PGD2, another major product of COX-2, to different types of receptors. While activation of the D-type prostanoid (DP) receptor (now named DP1 receptor) mediates the protective effect of prostaglandin D2 (PGD2), ${ }^{35}$ a chemoattractant receptor homologous molecule expressed on Th2 cells (CRTH2, now also termed DP2) promotes inflammation by facilitating chemotaxis of CRTH2-positive leukocytes to inflamed colon tissues. ${ }^{36}$ While immunohistochemical staining of DP1 remains constant, DP2 staining is strongly increased in colon tissues (mainly infiltrated leukocytes in lamina propria of the colonic mucosa and colon epithelia) of UC patients compared to those of healthy subjects. ${ }^{36}$ This finding together with our finding that COX-2 expression in the colonic epithelium is dramatically induced by mTORC1 activation, collectively suggest that besides Th17 cells recruitment, the proinflammatory effects of mTORC1-COX-2 in human UC and experimental UC may be also attributed to induction of the chemotaxis of leukocytes via preferential binding of overproduced PGD2 to the outnumbered DP2 receptor at the UC loci.

In summary, by combining data from two opposite mice genetic models and biopsies of UC patients, the current study suggests that colonic epithelial mTORC1 activity is critical for the pathogenesis of UC and establishes a novel communication link between colonic epithelium and immune cells during UC development (illustrated in Fig. 7). Furthermore, while colonic epithelial mTORC1 is not essential for normal colonic function, it promotes UC via COX-2-dependent activation and recruitment of the Th17 cells. Thus, the present study provides a new rationale for targeting colonic epithelial mTORC1 as a potential strategy to prevent or treat UC, effectively and safely. 
a
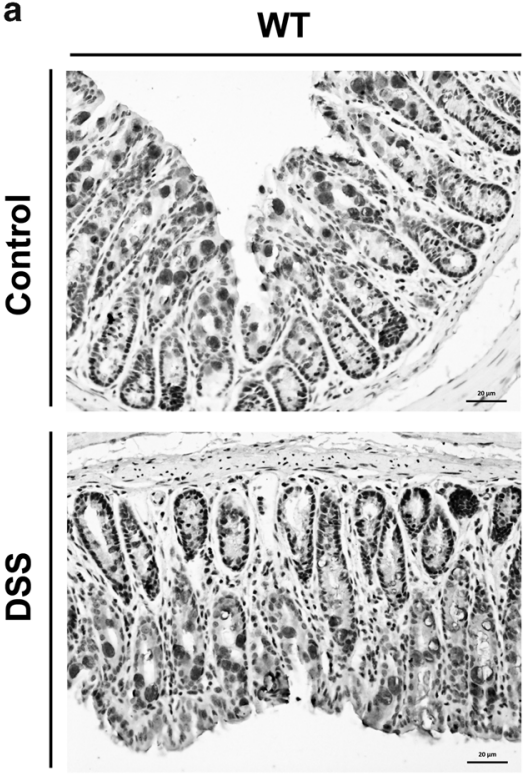

CEC-TSC1 KO
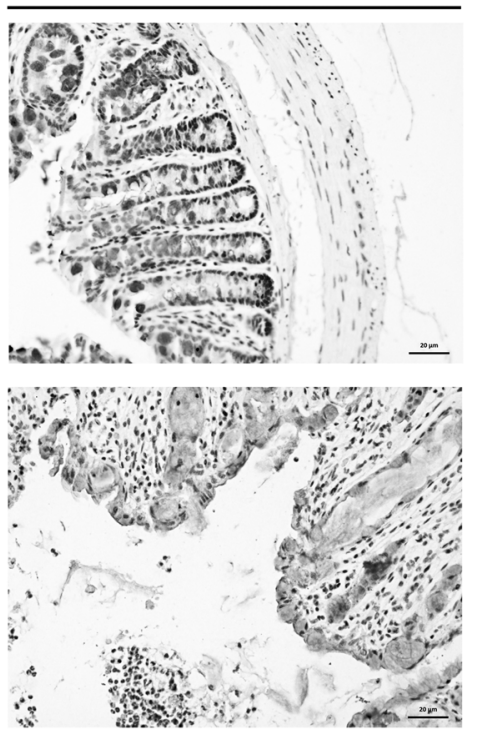

\section{Mice pair 2}

WT

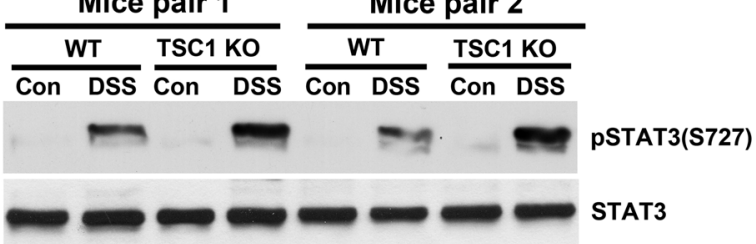

b

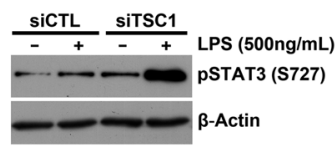

C

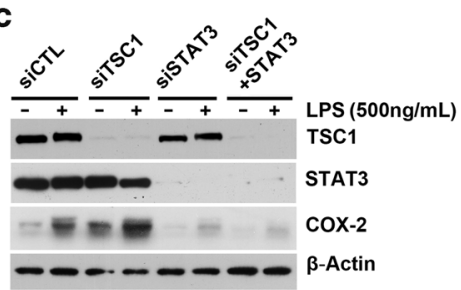

d

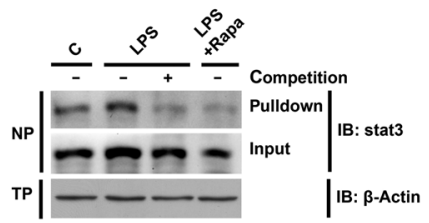

Fig. 5 mTORC1-mediated COX-2 transactivation is dependent on STAT3. a Control mice (WT) or mice with TSC1 ablation in colonic epithelial cells (CEC-TSC1 KO) were treated with or without DSS. STAT3 S727 phosphorylation in colonic epithelia was analyzed by immunohistochemical staining (upper) and western blotting (lower). Representative images from 6 to 8 mice were shown. b NCM460 cells transfected with control or TSC1 siRNA were treated with or without LPS for $24 \mathrm{~h}$, followed by observation of STAT3 phosphorylation (S727) using Western Blotting. c NCM460 cells transfected with a combination of TSC1 (or control) and STAT3 (or control) siRNA were treated as above, with expression of COX-2 protein assayed via Western Blotting. d NCM460 cells treated with a combination of LPS (or medium) and rapamycin (or medium) were analyzed for binding of STAT3 to COX-2 promoter, via a Streptavidin-Agarose DNA Pulldown assay. For competition, 20-fold excess non-labeled probe were used in the presence of biotin-labeled probe. NP nuclear protein, TP total protein. For Western Blotting and Streptavidin-Agarose DNA Pulldown assay, representative images from at least three independent experiments were shown

\section{MATERIALS AND METHODS}

Human biopsies

Patients with UC and non-UC control subjects were recruited with written informed consent for this study at the Digestive Division, Nanfang Hospital, Southern Medical University. The non-UC controls were patients who underwent screening colonoscopies without active gastrointestinal pathology. The diagnosis of UC was based on a standard combination of clinical, endoscopic, and histological criteria. The severity of macroscopic inflammation of the colonic mucosa at colonoscopy was graded according to the Mayo score for UC. ${ }^{37}$ The disease activity was evaluated according to the Rachmilewitz Clinical Activity Index. ${ }^{38}$

Generation of CA1-Cre;TSC1 ${ }^{\mathrm{FL} / \mathrm{FL}}$ and CA1-Cre;RPTOR ${ }^{\mathrm{FL} / \mathrm{FL}}$ mice Female floxed TSC1 mice ( $T S C 1^{\mathrm{FL} / F L}$ ) containing LoxP sites flanking exons 17 and 18 of the TSC1 gene or floxed RPTOR mice $\left(R P T O R^{\mathrm{FL} / \mathrm{FL}}\right)$ containing LoxP sites flanking alternate exon 1 and common exon 2 of the RPTOR gene were crossed with male carbonic anhydrase 1 (CA1)-Cre mice, whose expression of Cre recombinase is under control of the CA1 promoter and limited to the epithelial cells of the cecum and colon. The resulting CA1Cre; TSC $^{\mathrm{FL} / \mathrm{FL}}$ (CEC-TSC1 KO) or CA1-Cre; RPTOR ${ }^{\mathrm{FL} / \mathrm{FL}}$ (CEC-RPTOR KO) mice are hemizygous for the CA1-Cre transgene and homozygous for the floxed TSC1 or RPTOR allele. TSC $1^{\mathrm{FL} / \mathrm{FL}}$ or $R P T O R^{\mathrm{FL} / F \mathrm{~L}}$ cohoused littermates lacking the Cre gene were used as control (marked as WT in all experiments for convenience but were not exactly wild-type). All animals were backcrossed for 8 generations onto the C57BL/6 background. Experiments involving mice were performed in compliance with the Guide for the Care and Use of Laboratory Animals and protocols were approved by the Animal Care and Use Committee of Southern Medical University. 
a

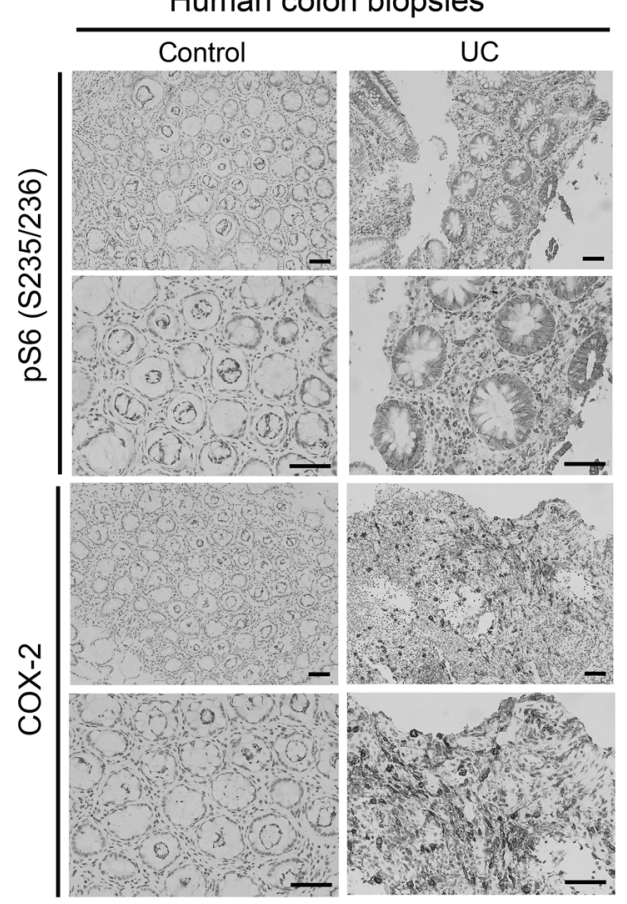

b
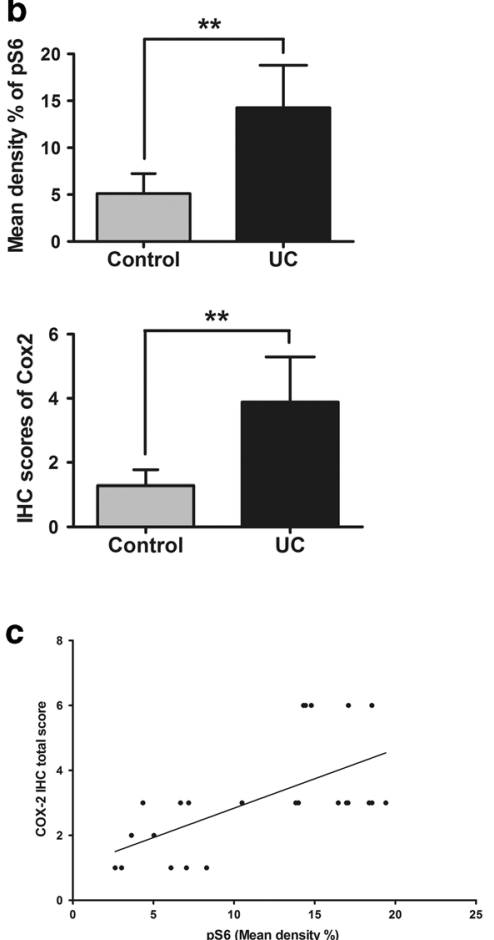

Fig. 6 mTORC1 activity and COX-2 expression are dramatically upregulated and positively correlated in patients with UC Colon biopsies from 7 healthy subjects and 17 patients with UC were first stained with hematoxylin, followed by staining for phosphorylated S6 (S235/236) or COX2, respectively. a Representative images of phosphorylated S6 (S235/236) staining (upper panel) or COX-2 staining (lower panel). Original magnification, $\times 20$ and $\times 40$ as indicated by the scale bars. Scale bars, $50 \mu \mathrm{m}$. b Statistical analysis of mean intensity $\%$ of S6 staining (upper panel) or IHC score of COX-2 staining (lower panel) of colon biopsies from these healthy subjects and UC patients. ${ }^{* *} P<0.01$. c Correlation of S6 phosphorylation (S235/236) status with COX-2 expression in colon biopsies from these healthy subjects and UC patients

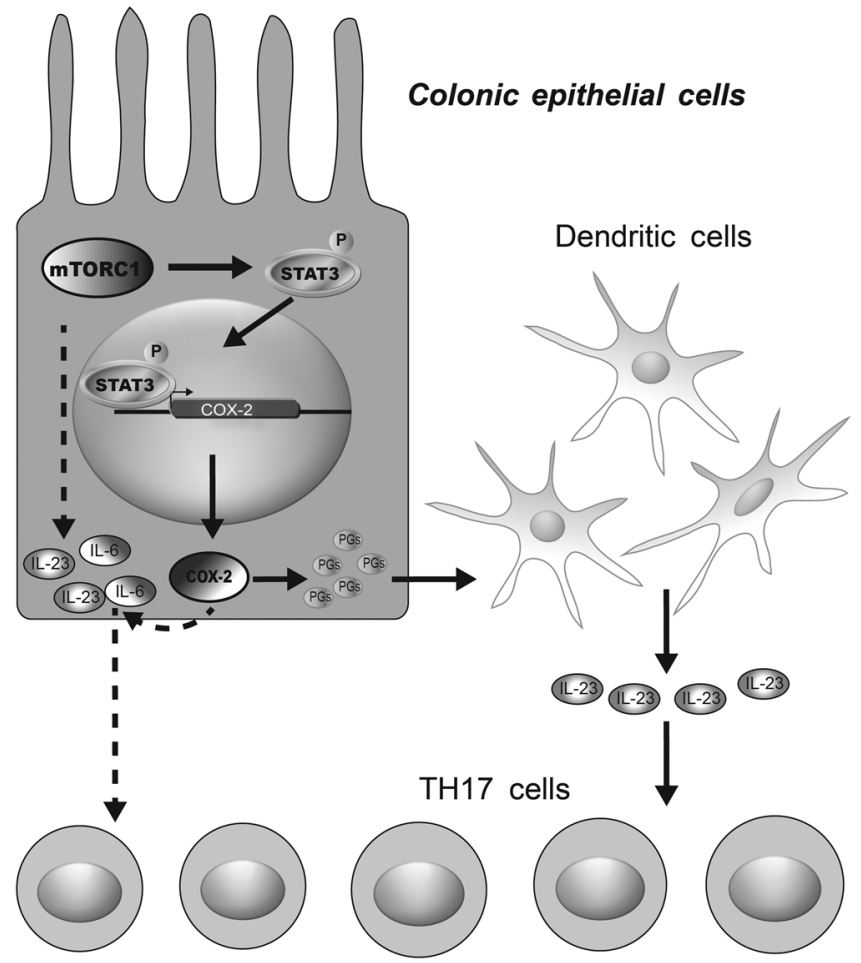

Fig. 7 Schematic diagram of the crosstalk between colonic epithelial cells and Th17 cells
Construction of the DSS-induced experimental colitis mice model and treatment

CA1-Cre; TSC1 ${ }^{\mathrm{FL} / \mathrm{FL}}$ and CA1-Cre; RPTOR ${ }^{\mathrm{FL} / \mathrm{FL}}$ mice and their control littermates (5-6 mice in each group) were weighed and mice 17-20 $\mathrm{g}$ were administered 3\% DSS dissolved in drinking water for 7 days. The DSS solution was renewed every 2 days. For rapamycin administration, mice were treated each day with vehicle control or rapamycin (1 mg/kg, Sigma, St Louis, MO) dissolved in water containing 5\% 1-methyl-2-pyrrolidinone (Sigma) and 15\% polyvinylpyrrolidone (Sigma) by gavage from the first day of DSS treatment to sacrifice. For celecoxib administration, mice were treated daily with vehicle control or celecoxib (Selleck Chemicals, Houston, TX) dissolved in water containing $0.5 \%$ methylcellulose (Sigma) from the first day of DSS treatment till final sacrifice. The dose used was $5 \mathrm{mg} / \mathrm{kg}$ for TSC1 KO mice and $2.5 \mathrm{mg} / \mathrm{kg}$ for WT mice. Body weight and Gl bleeding were monitored daily as described elsewhere. ${ }^{39}$ Disease activity index (DAI) and colonic damage scores were estimated as detailed previously. ${ }^{40}$ Histopathological scores were graded according to a previously published system. ${ }^{41}$

Isolation of colonic epithelial cells and LP lymphocytes

Colonic epithelial cells were isolated from freshly harvested colons using Percoll gradient centrifugation according to the procedure described previously. ${ }^{42}$ Briefly, colons were cut into $0.2 \times 0.2 \mathrm{~cm}$ segments and incubated in HBSS containing 5\% FBS, $1 \mathrm{mM} \mathrm{DTT}$, and $0.5 \mathrm{mM}$ EDTA ( $\mathrm{pH} 8.0$ ) at $4{ }^{\circ} \mathrm{C}$ for $1 \mathrm{~h}$ in an orbital shaker. The supernatant was filtered through a $250 \mu \mathrm{m}$ sieve, and epithelial cells were purified by centrifugation through a $25 / 40 \%$ discontinuous Percoll gradient. Predominantly epithelial cells were collected from the interface between the two layers and resuspended in $100 \%$ FCS. Typically, epithelial cells were $>95 \%$ pure. LP lymphocytes were isolated as described previously. ${ }^{43}$ 
Briefly, the colons were in Hank's Balanced Salt Solution (HBSS) supplemented with $4 \%$ FBS and $5 \mathrm{mM}$ EDTA (Sigma) at $37^{\circ} \mathrm{C}$ for $25 \mathrm{~min}$ to remove epithelial cells and intraepithelial lymphocytes. The colon fragments were then incubated with RPMI 1640 supplemented with $4 \%$ FBS and $1 \mathrm{mg} / \mathrm{ml}$ collagenase type IV (R\&D) and Dnase I (Sigma) at $37{ }^{\circ} \mathrm{C}$ for $25 \mathrm{~min}$ with stirring. The digested tissues were pooled together and separated on a $40 / 80 \%$ discontinuous Percoll gradient (GE Healthcare). After centrifugation at $2500 \mathrm{rpm}$ for $25 \mathrm{~min}$, LP mononuclear cells were collected at the interface of the Percoll gradient, washed, and suspended in RPMI 1640 containing 4\% FBS.

mRNA expression array

Total RNA was extracted from colonic epithelial cells pooled from 3 mice using TRIzol reagent and submitted to Shanghai Biotechnology Corporation for hybridization on a Whole Mouse Genome $4 \times 44$ K Microarray (014868, Agilent technologies, Santa Clara, CA). For RNA amplification and labeling, total RNA was amplified and labeled using a Low Input Quick Amp Labeling Kit, One-Color (Cat\#5190-2305, Agilent technologies, Santa Clara, CA, US), following the manufacturer's instructions. Labeled cRNA were purified with an RNeasy mini kit (Cat\#74106, QIAGEN, GmBH, Germany). For hybridization, each slide was hybridized with 1.65 $\mu \mathrm{g}$ Cy3-labeled cRNA using a Gene Expression Hybridization Kit (Cat\#5188-5242, Agilent) in a Hybridization Oven (Cat\#G2545A, Agilent), according to the manufacturer's instructions. After $17 \mathrm{~h}$ of hybridization, slides were washed in staining dishes (Cat\#121, Thermo Shandon, Waltham, MA, US) with a Gene Expression Wash Buffer Kit (Cat\#5188-5327, Agilent), following the manufacturer's instructions. Slides were scanned by an Agilent Microarray Scanner (Cat\#G2565CA, Agilent) with the following default settings: dye channel, green; scan resolution, $5 \mu \mathrm{m}$; PMT $100 \%$, $10 \%, 16$ bit. For data acquisition, data were extracted with Feature Extraction software 10.7 (Agilent). Raw data were normalized by the Quantile algorithm, Gene Spring Software 11.0 (Agilent). All the raw data and metadata are available at http://www.ncbi.nlm.nih.gov/geo/query/acc.cgi?token=yjohsywyr dsdhor\&acc $=$ GSE74674.

Histology and immunohistochemical staining

Colon biopsies were fixed overnight with $4 \%$ formaldehyde in PBS (pH 7.2), processed, and embedded in paraffin wax. Tissues were

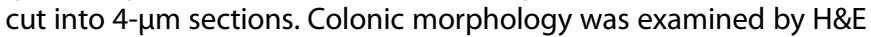
staining. To localize phosphorylated S6 (S235/S236) and COX2 expression, sections were stained with anti-phosphorylated S6 (1:200, Cell Signaling, Danvers, MA) or anti-COX2 (1:100, Cell Signaling) antibodies as primary antibodies overnight at $4{ }^{\circ} \mathrm{C}$, followed by staining with rabbit horseradish peroxidaseconjugated anti-lgG as second antibody at room temperature for $1 \mathrm{~h}$. Antigens were then visualized with 3,3'-diaminobenzidine substrate (Sigma) and observed and photographed on an Olympus BX51 microscope. Quantitative analysis of the immunostained images of human biopsies was performed by positive cell number counting and computerized optical density (OD) measurements with Image Pro Plus 6.0 software (Media Cybernetics, MD, USA). Positively stained regions of phosphorylated S6 (S235/S236) in the image were selected by HSI (hue, saturation and lightness) with $\mathrm{S}$ from 0 to 255 , I from 0 to 230 and $\mathrm{H}$ from 0 to 30 , and the brown color was then converted into a grayscale signal. The grayscale signal was measured as mean optical intensity of staining (mean density) within the tissue masks. Each image was assigned a score calculated by multiplying the staining intensity by the area of positively stained cells.

\section{Flow cytometry}

To detect Th17 cells, isolated lamina propria cells were first stained with anti-CD4 (eBioscience, San Diego, CA). After fixation and permeabilization, cells were further stained intracellularly with
anti-RORyt (eBioscience). Flow cytometric analysis was performed on a CytoFLEX (Beckman). The results were evaluated with CytExpert 1.2.8.0 software.

\section{Indirect immunofluorescence}

Tissue samples were fixed and the slides were incubated with $1 \%$ BSA for $1 \mathrm{~h}$, followed by incubation with unconjugated rabbit antiIL-17 (1:100, Abcam, Cambridge, MA) and mouse anti-CD4 (1:100, Boster, Wuhan, China) antibodies overnight at $4{ }^{\circ} \mathrm{C}$. The slides were then incubated with Alexa 594-conjugated anti-rabbit and Alexa 488-conjugated anti-mouse secondary antibodies (Invitrogen) for an additional hour. After incubation, the sections were covered with ProLong ${ }^{\circledR}$ Gold antifade reagent with 4',6-diamidino2-phenylindole (DAPI, Invitrogen) and analyzed using an Olympus FV1000 confocal fluorescence microscope, equipped with a Hamamatsu camera. Red filters (range $575-615 \mathrm{~nm}$ ) and green filters (range $500-550 \mathrm{~nm}$ ) were used for Alexa 594 and Alexa 488 staining, respectively.

Polymerase chain reaction (PCR)

Total RNA was extracted using the TRIzol reagent (Invitrogen, Grand Island, NY). For genotyping, conventional PCR was carried out in a BioRad DNA Engine. For quantitative reverse transcription PCR (RT-qPCR), first-strand cDNAs were synthesized using a PrimeScript $^{\circledR}$ 1st Strand cDNA Synthesis Kit (TaKaRa, Shiga, Japan). Quantitative PCR was performed in a LightCycler $^{\circledR}$ Nano SW 1.1 system (Roche) using SYBR green PCR reagent kits (TaKaRa). The relative amount of transcripts was calculated using the $2^{-\Delta \Delta C t}$ formula. PCR primers are provided in Table S1.

\section{Western blotting}

Cells and tissues were lysed by $2 \%$ sodium dodecyl sulfate with $10 \%$ glycerol, $10 \mathrm{mM}$ Tris- $\mathrm{HCl}$ (pH 6.8), $10 \mathrm{mM}$ dithiothreitol and 1 $\mathrm{mM}$ phenylmethylsulfonyl fluoride. The lysates were centrifuged and the supernatants were separated by SDS-PAGE and blotted onto a nitrocellulose (NC) membrane (Bio-Rad Laboratories, Hercules, CA). Membranes were blocked in 5\% non-fat milk in TBS for $1 \mathrm{~h}$ at room temperature, and then incubated with primary antibodies overnight at $4{ }^{\circ} \mathrm{C}$, followed by washing in TBS containing $0.1 \%$ Tween 20 and incubation with an HRP-conjugated secondary antibody for $1 \mathrm{~h}$ at room temperature. Bands were visualized by enhanced chemiluminescence (ECL Kit, Amersham Biosciences, Piscataway, NJ).

Streptavidin-agarose DNA pulldown assay

The biotinylated primers listed in S1 Table were synthesized and purified through HPLC by Life Technologies (Shanghai, China). These primers were used to prepare a 479-bp double-stranded biotinylated COX-2 promoter probes. None-labeled primers were also used to prepare a probe of equal length for competition control. The whole pulldown procedure was strictly following the protocols suggested by the literature. ${ }^{44}$

\section{Statistical analysis}

All statistical analyses were performed using SPSS 13.0 software. Data are presented as mean values \pm s.e.m. of at least three independent experiments, and analyzed using the two-tailed $t$ test or one-way ANOVA with multiple comparisons, followed by Bonferroni post-hoc test for significance. A $p$-value $<0.05$ was considered statistically significant.

\section{ACKNOWLEDGEMENTS}

We acknowledge funding from the State Key Development Program for Basic Research of China (2013CB945203, 2015CB55360), National Natural Sciences Foundation of China (U1301222, 81530070, 31600941, 81301525, and 81372136), and GDHVPS (2011). 


\section{AUTHOR CONTRIBUTIONS}

J.Y., S.Y., C.J., Z.Z., and B.X. designed research; L.X., Z.L., S.Q., H.M., D.X., J.C., and H.Z. performed experiments; L.X., H.M., Z.Z., and B.X. analyzed the microarray data; L.X., L. M., L.H., C.J., Z.Z., and B.X. contributed to data analysis; Z.Z., B.X., and L.H. wrote the paper; Z.H., C.J., Z.Z. and B.X. contributed to study supervision; H.Z. and L.S. collected colon biopsies from healthy subjects and patients with ulcerative colitis.

\section{ADDITIONAL INFORMATION}

The online version of this article (https://doi.org/10.1038/s41385-018-0018-3) contains supplementary material, which is available to authorized users.

Competing interest: The authors declare no competing interests.

Publisher's note: Springer Nature remains neutral with regard to jurisdictional claims in published maps and institutional affiliations.

\section{REFERENCES}

1. Kaplan, G. G. The global burden of IBD: from 2015 to 2025. Nat. Rev. Gastroenterol. Hepatol. 12, 720-727 (2015).

2. Abraham, C. \& Cho, J. H. Inflammatory bowel disease. N. Engl. J. Med. 361, 2066-2078 (2009).

3. Kobayashi, T. et al. IL 23 differentially regulates the Th1/Th17 balance in ulcerative colitis and Crohn's disease. Gut 57, 1682-1689 (2008).

4. Galvez, J. Role of Th17 cells in the pathogenesis of human IBD. ISRN Inflamm. 2014, 928461 (2014).

5. Dong, C. TH17 cells in development: an updated view of their molecular identity and genetic programming. Nat. Rev. Immunol. 8, 337-348 (2008).

6. Bettelli, E., Oukka, M. \& Kuchroo, V. K. T(H)-17cells in the circle of immunity and autoimmunity. Nat. Immunol. 8, 345-350 (2007).

7. Siakavellas, S. I. \& Bamias, G. Role of the IL-23/IL-17 axis in Crohn's disease. Discov. Med. 14, 253-262 (2012).

8. Gulen, M. F. et al. Inactivation of the enzyme GSK3alpha by the kinase IKK promotes AKT-mTOR signaling pathway that mediates interleukin-1-induced Th17 cell maintenance. Immunity 37, 800-812 (2012).

9. Gao, S. et al. The activation of mTOR is required for monocyte pro-inflammatory response in patients with coronary artery disease. Clin. Sci. 128, 517-526 (2015)

10. Kim, T. H., Choi, S. J., Lee, Y. H., Song, G. G. \& Ji, J. D. Combined therapeutic application of mTOR inhibitor and vitamin $\mathrm{D}(3)$ for inflammatory bone destruction of rheumatoid arthritis. Med. Hypotheses 79, 757-760 (2012).

11. Dos Santos, S., Delattre, A. I., De Longueville, F., Bult, H. \& Raes, M. Gene expression profiling of LPS-stimulated murine macrophages and role of the NFkappaB and PI3K/mTOR signaling pathways. Ann. N. Y. Acad. Sci. 1096, 70-77 (2007).

12. Hu, S. et al. mTOR inhibition attenuates dextran sulfate sodium-induced colitis by suppressing $\mathrm{T}$ cell proliferation and balancing TH1/TH17/Treg profile. PLoS One 11, e0154564 (2016).

13. Bhonde, M. R. et al. A novel mTOR inhibitor is efficacious in a murine model of colitis. Am. J. Physiol. Gastrointest. Liver. Physiol. 295, G1237-G1245 (2008).

14. Farkas, S. et al. Rapamycin decreases leukocyte migration in vivo and effectively reduces experimentally induced chronic colitis. Int. J. Colorectal Dis. 21, 747-753 (2006).

15. Laplante, M. \& Sabatini, D. M. mTOR signaling in growth control and disease. Cell 149, 274-293 (2012).

16. Xue, Y., Johnson, R., Desmet, M., Snyder, P. W. \& Fleet, J. C. Generation of a transgenic mouse for colorectal cancer research with intestinal cre expression limited to the large intestine. Mol. Cancer Res. 8, 1095-1104 (2010).

17. Samad, T. A. et al. Interleukin-1beta-mediated induction of Cox-2 in the CNS contributes to inflammatory pain hypersensitivity. Nature 410, 471-475 (2001).

18. Tsuzaki, M. et al. IL-1 beta induces COX2, MMP-1, -3 and -13, ADAMTS-4, IL-1 beta and IL-6 in human tendon cells. J. Orthop. Res. 21, 256-264 (2003).

19. Dawn, B. et al. IL-6 plays an obligatory role in late preconditioning via JAK-STAT signaling and upregulation of iNOS and COX-2. Cardiovasc. Res. 64, 61-71 (2004).
20. Anderson, G. D. et al. Selective inhibition of cyclooxygenase (COX)-2 reverses inflammation and expression of COX-2 and interleukin 6 in rat adjuvant arthritis. J. Clin. Invest. 97, 2672-2679 (1996).

21. Hsu, C. K., Lee, I. T., Lin, C. C., Hsiao, L. D. \& Yang, C. M. Sphingosine-1-phosphate mediates COX-2 expression and PGE2 /IL-6 secretion via C-Src-dependent AP-1 activation. J. Cell. Physiol. 230, 702-715 (2015).

22. Nair, R. P. et al. Genome-wide scan reveals association of psoriasis with IL-23 and NF-kappaB pathways. Nat. Genet. 41, 199-204 (2009).

23. Sheibanie, A. F. et al. The proinflammatory effect of prostaglandin E2 in experimental inflammatory bowel disease is mediated through the IL-23-->IL-17 axis. J. Immunol. 178, 8138-8147 (2007).

24. Khayrullina, T., Yen, J. H., Jing, H. \& Ganea, D. In vitro differentiation of dendritic cells in the presence of prostaglandin E2 alters the IL-12/IL-23 balance and promotes differentiation of Th17 cells. J. Immunol. 181, 721-735 (2008).

25. Sano, T. et al. An IL-23R/IL-22 circuit regulates epithelial serum amyloid A to promote local effector Th17 responses. Cell 164, 324 (2016).

26. Lo, H. W., Cao, X., Zhu, H. \& Ali-Osman, F. Cyclooxygenase-2 is a novel transcriptional target of the nuclear EGFR-STAT3 and EGFRvIll-STAT3 signaling axes. Mol. Cancer Res. 8, 232-245 (2010).

27. Huang, B. et al. mTORC1 prevents preosteoblast differentiation through the Notch signaling pathway. PLoS Genet. 11, e1005426 (2015).

28. Simon, L. S. Role and regulation of cyclooxygenase-2 during inflammation. Am. J. Med. 106, 37S-42S (1999).

29. Paiotti, A. P. et al. The role of nonsteroidal antiinflammatory drugs and cyclooxygenase-2 inhibitors on experimental colitis. In Vivo 26, 381-393 (2012).

30. Martin, A. R., Villegas, I. \& Alarcon de la Lastra, C. The COX-2 inhibitor, rofecoxib, ameliorates dextran sulphate sodium induced colitis in mice. Inflamm. Res. 54, 145-151 (2005).

31. Dannhardt, G. \& Kiefer, W. Cyclooxygenase inhibitors-current status and future prospects. Eur. J. Med. Chem. 36, 109-126 (2001).

32. Mahadevan, U., Loftus, E. V. Jr., Tremaine, W. J. \& Sandborn, W. J. Safety of selective cyclooxygenase-2 inhibitors in inflammatory bowel disease. Am. J. Gastroenterol. 97, 910-914 (2002).

33. El-Medany, A., Mahgoub, A., Mustafa, A., Arafa, M. \& Morsi, M. The effects of selective cyclooxygenase-2 inhibitors, celecoxib and rofecoxib, on experimental colitis induced by acetic acid in rats. Eur. J. Pharmacol. 507, 291-299 (2005).

34. Okayama, M. et al. Aggravation by selective COX-1 and COX-2 inhibitors of dextran sulfate sodium (DSS)-induced colon lesions in rats. Dig. Dis. Sci. 52, 2095-2103 (2007).

35. Ajuebor, M. N., Singh, A. \& Wallace, J. L. Cyclooxygenase-2-derived prostaglandin $\mathrm{D}(2)$ is an early anti-inflammatory signal in experimental colitis. Am. J. Physiol. Gastrointest. Liver Physiol. 279, G238-G244 (2000).

36. Sturm, E. M. et al. Opposing roles of prostaglandin D2 receptors in ulcerative colitis. J. Immunol. 193, 827-839 (2014).

37. Cooney, R. M., Warren, B. F., Altman, D. G., Abreu, M. T. \& Travis, S. P. Outcome measurement in clinical trials for Ulcerative Colitis: towards standardisation. Trials 8, 17 (2007).

38. Takahashi, M. et al. An inverse correlation of human peripheral blood regulatory $T$ cell frequency with the disease activity of ulcerative colitis. Dig. Dis. Sci. 51 677-686 (2006).

39. Cooper, H. S., Murthy, S. N., Shah, R. S. \& Sedergran, D. J. Clinicopathologic study of dextran sulfate sodium experimental murine colitis. Lab Invest. 69, 238-249 (1993).

40. Ohkusa, T. Production of experimental ulcerative colitis in hamsters by dextran sulfate sodium and changes in intestinal microflora. Nihon Shokakibyo Gakkai zasshi 82, 1327-1336 (1985).

41. Ohkawara, T. et al. Amelioration of dextran sulfate sodium-induced colitis by antimacrophage migration inhibitory factor antibody in mice. Gastroenterology 123, 256-270 (2002).

42. Denning, T. L. et al. Expression of IL-10 receptors on epithelial cells from the murine small and large intestine. Int. Immunol. 12, 133-139 (2000).

43. Atarashi, K. et al. ATP drives lamina propria $\mathrm{T}(\mathrm{H}) 17$ cell differentiation. Nature $\mathbf{4 5 5}$, 808-812 (2008).

44. Wu, K. K. Analysis of protein-DNA binding by streptavidin-agarose pulldown. Methods Mol. Biol. 338, 281-290 (2006). 